Draft version November 20, 2018

Preprint typeset using $\mathrm{LAT}_{\mathrm{E}} \mathrm{X}$ style emulateapj v. 11/10/09

\title{
RADIATIVE AND KINETIC FEEDBACK BY LOW-MASS PRIMORDIAL STARS
}

\author{
Daniel Whalen ${ }^{1,2}$, Robert M. Hueckstaedt ${ }^{3} \&$ Thomas O. McConkie ${ }^{2,4}$ \\ Draft version November 20, 2018
}

\begin{abstract}
Ionizing UV radiation and supernova flows amidst clustered minihalos at high redshift regulated the rise of the first stellar populations in the universe. Previous studies have addressed the effects of very massive primordial stars on the collapse of nearby halos into new stars, but the absence of the odd-even nucleosynthetic signature of pair-instability supernovae in ancient metal-poor stars suggests that Population III stars may have been less than $100 \mathrm{M}_{\odot}$. We extend our earlier survey of local UV feedback on star formation to $25-80 \mathrm{M}_{\odot}$ stars and include kinetic feedback by supernovae for $25-40 \mathrm{M}_{\odot}$ stars. We find radiative feedback to be relatively uniform over this mass range, primarily because the larger fluxes of more massive stars are offset by their shorter lifetimes. Our models demonstrate that prior to the rise of global UV backgrounds, Lyman-Werner photons from nearby stars cannot prevent halos from forming new stars. These calculations also reveal that violent dynamical instabilities can erupt in the UV radiation front enveloping a primordial halo but that they ultimately have no effect on the formation of a star. Finally, our simulations suggest that relic H II regions surrounding partially evaporated halos may expel Lyman-Werner backgrounds at lower redshifts, allowing stars to form that were previously suppressed. We provide fits to radiative and kinetic feedback on star formation for use in both semianalytic models and numerical simulations.

Subject headings: cosmology: theory - early universe - galaxies: high redshift - H II regionsintergalactic medium - radiative transfer
\end{abstract}

\section{INTRODUCTION}

The survival of cosmological minihalos in the ionizing and Lyman-Werner (LW) UV fields of primordial stars is key to the rise of stellar populations at high redshifts. Numerical models suggest that the first stars are very massive, $25-500 \mathrm{M}_{\odot}$, and that they form in isolation in small dark matter halos of $\sim 10^{5}-10^{7} \mathrm{M}_{\odot}$ at $z \sim 20$ - 30 (Bromm et al. 1999, 2002; Nakamura \& Umemura 2001; Abel et al. 2000, 2002; O'Shea \& Norman 2007). These stars create large H II regions $2.5-5 \mathrm{kpc}$ in radius that can engulf nearby halos (Whalen et al. 2004; Kitavama et al. 2004; Alvarez et al. 2006; Abel et al. 2007; Wise \& Abel 2008a). From z 10 - 20 Population III (Pop III) stars also build up a global LW background that sterilizes minihalos of $\mathrm{H}_{2}$, delaying or preventing the formation of new stars (Haiman et al. 1997, 2000; Machacek et al. 2001; Mesinger et al. 2006; Wise \& Abel 2007; Susa 2007; O'Shea \& Norman 2008; Wise \& Abel 2007; Johnson et al. 2007; Mesinger et al. 2009; Ahn et al. 2009). At high redshifts, ionizing radiation is relatively local while LW photons can originate from many megaparsecs away because their energies lie below the ionization limit of $\mathrm{H}$.

The picture is simpler in the first generation, in which there is no LW background. Simulations of halo photoevaporation by nearby very massive ( $\gtrsim 100 \mathrm{M}_{\odot}$ ) primordial stars have been performed, both with (O'Shea et al. 2005; Susa \& Umemura 2006;

\footnotetext{
${ }^{1}$ McWilliams Fellow, Department of Physics, Carnegie Mellon University, Pittsburgh, PA 15213. Email: dwhalen@lanl.gov

2 Nuclear \& Particle Physics, Astrophysics, and Cosmology (T-2), Los Alamos National Laboratory, Los Alamos, NM 87545

3 Applied Physics (X-2), Los Alamos National Laboratory

${ }^{4}$ Department of Physics and Astronomy, Brigham Young University, Provo, UT 84602
}

Ahn \& Shapiro 2007; Whalen et al. 2008a; Wise \& Abel 2008b; Hasegawa et al.|2009; Susa et al.|2009) and without (Shapiro et al. 2004; Iliev et al. 2005) $\mathrm{H}_{2}$ gas chemistry. The two- and three-dimensional studies are in good agreement with each other but not with the onedimensional studies (Ahn \& Shapiro 2007), primarily due to the serious hydrodynamical artifacts that arise from unphysical shock focusing and central bounce that occur in one-dimensional spherical coordinate meshes. When multifrequency photon transport is coupled to primordial chemistry and hydrodynamics, halo photoevaporation proceeds in two stages. First, LW photons from the star reach the halo before the I-front, partly dissociating molecular hydrogen in its core and slowing or halting its collapse. Not all of the $\mathrm{H}_{2}$ is erradicated because molecular hydrogen deep in the halo partly shields itself from the photons and small free electron fractions restore $\mathrm{H}_{2}$ by catalysis through the $\mathrm{H}^{-}$channel. The I-front decelerates as it approaches the halo and transforms from R-type to D-type. Molecular hydrogen forms in the small ionized fractions and warm temperatures in the outer layers of the front (e.g. Ricotti et al. 2001; Whalen \& Norman 2008a), partly shielding the interior of the halo from LW photons from the star and even allowing $\mathrm{H}_{2}$ fractions in the core to rise in some cases. The ionization front preferentially advances through the low densities above and below the halo and assumes a cometary shape, with the inner regions of the halo casting a shadow.

Second, after the death of the star the H II region surrounding the halo begins to recombine out of equilibrium, rapidly forming $\mathrm{H}_{2}$ and $\mathrm{HD}$ that can cool the ionized gas down to the CMB temperature (Nakamura \& Umemura 2002; Johnson \& Bromm 2006; Yoshida et al.|2007; Greif et al.|2008). At the same time, 
$\mathrm{H}_{2}$ reconstitutes in the core, rapidly surpassing its original levels and allowing gas to again cool and collapse. Meanwhile, the I-front shock remnant continues to converge on the halo core from one side while warm ionized gas presses down into the shadow and wraps around the halo from behind. If the UV flux from the star is not too high, the relic shock compresses the core and enriches it with the $\mathrm{H}_{2}$ originally formed in the I-front, accelerating its cooling and collapse into a new star. If the flux from the star is large, the ionized gas instead disrupts the core and pre-empts new star formation. The studies performed to date assume stars that do not die in supernova (SN) explosions and therefore exclude ram pressure stripping of the halo by the remnant (Cen \& Riquelme 2008; Sakuma \& Susa 2009) and its contamination and cooling by heavy elements. In the first generation of stars, both stages of halo photoevaporation occur on time scales that are short in comparison to merger or accretion times.

In large scale calculations of cosmological structure formation, halo evaporation is usually modeled with metagalactic ionizing and LW backgrounds that uniformly permeate the simulation volume. Such halos evolve very differently than when photon transport is performed because they are photoevaporated and photodissociated from the inside out. Rather than being compressed and shielded from LW photons, baryons are expelled from all directions. Consequently, radiative feedback is invariably negative in these models. An exception to this are the recent simulations by Mesinger et al. (2009), who find that low mass halos that are easily evaporated at early times later pool gas and form stars when the uniform fields are turned off and the fossil H II regions cool down. Self-shielding of $\mathrm{H}_{2}$ from external UV sources cannot be modeled by imposed backgrounds, so molecular hydrogen is driven to much lower levels in these halos than in real ones. In general, negative feedback in studies that rely on metagalactic UV backgrounds should be taken as a (possibly extreme) upper limit. Star formation in the UV environments of the early universe is likely to be significantly more robust.

In principle, the parameter space for local radiative feedback between clustered minihalos is vast even if supernova effects are excluded, making the formulation of general rules for use in analytical models problematic. Halo mass, central baryon density, distance and luminosity of the star, stellar lifetime and spectral profile all govern halo evaporation. Distance and luminosity can be combined in a single parameter over intervals in stellar mass for which the shape of the source spectrum does not vary much. Whalen et al. (2008a) further reduced this parameter space by examining feedback in the smallest halo ever found to form a star in an adaptive mesh refinement (AMR) calculation but at four stages of collapse. Since more massive halos at the same central baryon density would be less affected by radiation, their findings constitute a conservative upper limit to the influence one star can exert on any neighbor halo capable of forming a star.

In this paper we extend our previous survey by examining the evaporation of minihalos by low-mass primordial stars prior to the buildup of a large LW background. Pop III stars from $25-80 \mathrm{M}_{\odot}$ have spectra with larger $\mathrm{LW}$ /ionizing UV ratios than those of the $120 \mathrm{M}_{\odot}$ star in our previous study and they illuminate other halos for longer times. We sample the same halo and central gas densities as in our earlier study in order to place upper limits on the radiative feedback of such stars on more massive halos. By determining the final state of the halo core several Myr after the death of the star we construct rules for local radiative and kinetic feedback as a function of stellar mass, initial core density, and distance to the star. In $\S 2$ we review our numerical methods, in $\S 3$ we describe our cosmological halo models, in $\S 4$ we tabulate both radiative and kinetic feedback in a photoevaporated halo, and in $\S 5$ we conclude.

\section{NUMERICAL ALGORITHM}

We perform our halo photoevaporation calculations with ZEUS-MP (Haves et al. 2006), a massively-parallel Eulerian astrophysical hydrodynamics code that solves the equations of ideal fluid dynamics:

$$
\begin{aligned}
\frac{\partial \rho}{\partial t} & =-\nabla \cdot(\rho \mathbf{v}) \\
\frac{\partial \rho v_{i}}{\partial t} & =-\nabla \cdot\left(\rho v_{i} \mathbf{v}\right)-\nabla p-\rho \nabla \Phi-\nabla \cdot \mathbf{Q} \\
\frac{\partial e}{\partial t} & =-\nabla \cdot(e \mathbf{v})-p \nabla \cdot \mathbf{v}-\mathbf{Q}: \nabla \mathbf{v} .
\end{aligned}
$$

Here, $\rho, e$, and the $v_{i}$ are the mass density, internal energy density, and velocity at each mesh point and $p=(\gamma-1) e$ and $\mathbf{Q}$ are the gas pressure and the von Neumann-Richtmeyer artificial viscosity tensor (Stone \& Norman 1992). ZEUS-MP evolves these equations with a second-order accurate monotonic advection scheme (van Leer 1977) in one, two, or three dimensions on Cartesian (XYZ), cylindrical (ZRP), or spherical polar (RTP) coordinate meshes. Our augmented version of the publically-available code self-consistently couples primordial gas chemistry (Whalen \& Norman 2006, $2008 \mathrm{~b}$ ) and multifrequency photon-conserving UV radiative transfer (Whalen \& Norman 2008a) to fluid dynamics for radiation hydroynamical transport of cosmological I-fronts.

\subsection{Primordial $H$ and He Chemistry}

We evolve $\mathrm{H}, \mathrm{H}^{+}, \mathrm{He}, \mathrm{He}^{+}, \mathrm{He}^{2+}, \mathrm{H}^{-}, \mathrm{H}_{2}^{+}, \mathrm{H}_{2}$, and $\mathrm{e}^{-}$with nine additional continuity equations and the nonequilibrium rate equations of Anninos et al. (1997):

$$
\frac{\partial \rho_{i}}{\partial t}=-\nabla \cdot\left(\rho_{i} \mathbf{v}\right)+\sum_{j} \sum_{k} \beta_{j k}(T) \rho_{j} \rho_{k}-\sum_{j} \kappa_{j} \rho_{j},
$$

where $\beta_{j k}$ is the rate coefficient of the reaction between species $\mathrm{j}$ and $\mathrm{k}$ that creates $(+)$ or destroys (-) species $\mathrm{i}$, and the $\kappa_{j}$ are the radiative rate coefficients. We assume that the species share a common velocity distribution. Mass and charge conservation, which are not guaranteed by either chemical or advective updates, are enforced each time the fluid equations are solved. The divergence terms and reaction network are operator-split and evolved on their respective time scales, as we explain in greater detail below.

${ }^{5}$ http://lca.ucsd.edu/portal/codes/zeusmp2 
Microphysical heating and cooling due to photoionization and gas chemistry is coupled to the gas energy density by an isochoric update that is operator-split from updates to the fluid equations:

$$
\dot{e}_{\text {gas }}=\Gamma-\Lambda \text {, }
$$

where $\Gamma$ is the cumulative heating rate due to photons of all frequencies and $\Lambda$ is the sum of the cooling rates due to collisional ionization and excitation of $\mathrm{H}$ and $\mathrm{He}$, recombinations of $\mathrm{H}$ and $\mathrm{He}$, inverse Compton scattering (IC) off the CMB, bremsstrahlung emission, and $\mathrm{H}_{2}$ cooling (Galli \& Palla 1998).

\subsection{Radiative Transfer}

Our photon-conserving UV transport Abel et al. 1999; Mellema et al. 2006), which is distinct from the flux-limited diffusion native to the public release of ZEUS-MP, solves the static approximation to the equation of transfer in flux form to compute radiative rate coefficients for the reaction network at every point on the coordinate mesh (Abel et al. 1999). As currently implemented, our code can transport photons from a point source centered in a spherical grid or in plane waves along the $x$ or $z$-axes of Cartesian or cylindrical meshes. In our models, the radiation from the star is treated as a plane wave that propagates along the positive $z$-axis. Plane waves are a good approximation to the UV ionizing flux at the interhalo separations in our study, but we attenuate their intensity by $1 / R^{2}$ to account for geometrical dilution.

As in Whalen et al. (2008a), we discretize the blackbody photon emission rates of the stars in our survey with 40 uniform bins from 0.755 to $13.6 \mathrm{eV}$ and $80 \mathrm{log}$ arithmically spaced bins from $13.6 \mathrm{eV}$ to $90 \mathrm{eV}$, again normalizing them by the total ionizing photon rates for Pop III stars by Schaerer (2002). The radiative reactions in our models are listed in Table 1 of Whalen \& Norman (2008a). We do not evaluate $\mathrm{H}_{2}$ photodissociation rates with radiative transfer. Instead, we calculate them along rays parallel to the direction of radiation flow using selfshielding functions modified for thermal broadening as prescribed by Draine \& Bertoldi (1996) to approximate the effects of gas motion. They are shown in equations 9 and 10 of Whalen \& Norman (2008a).

\subsection{Radiation Forces}

Since our prior survey of radiative feedback, we have implemented momentum deposition in the gas due to ionizations. Radiation pressure in ionizing UV transport comes into play at two locations: at the I-front itself and in recombining gas in the $\mathrm{H}$ II region. Whalen \& Norman (2006) examined the acceleration of fluid elements at the front and found that it was large but momentary, and that its inclusion alters the velocity of the front by only $1-2 \mathrm{~km} \mathrm{~s}^{-1}$. Direct momentum deposition within the $\mathrm{H}$ II region is only prominent where gas is very dense, like the center of a cosmological minihalo being evaporated by a star at its center. There, rapid successive cycles of ionization and recombination can impart radiation forces to the gas that are hundreds of times the strength of gravity at early times (lower left panel of Figure 1 in Kitayama et al. 2004). As these forces propel gas near the center of the halo out into the $\mathrm{H}$ II region, its densities and recombination rates fall, so more ionizing photons from the star reach the I-front. This higher flux results in I-fronts that are faster than when such forces are not included. Thus, in early UV breakout, radiation forces should speed up the I-front, and in trial runs we find that D-type fronts are $10-20 \%$ faster than when momentum transfer due ionizations is neglected. However, this effect is transient: after the internal rearrangement of gas deep within the $\mathrm{H}$ II region dilutes its interior, radiation forces there sharply fall.

We expect much weaker forces in this study because the front climbs a density gradient as it approaches the halo rather than descending one, so the gas behind the I-front is always relatively diffuse. Since new ionizations due to recombinations are far less frequent, less momentum will be imparted to the gas, so we include these effects only for completeness. Updates to the gas velocities are straightforward since momentum deposition due to direct photons from the source is always parallel to the direction of radiation flow. We describe the time scales on which momentum updates are performed below.

\subsection{Adaptive Subcycling}

A hierarchy of highly disparate characteristic time scales arises when gas dynamics, radiative transfer, and primordial chemistry are solved in a given application. The three governing times are the Courant time, the chemical time

$$
t_{\text {chem }}=0.1 \frac{n_{e}+0.001 n_{H}}{\dot{n}_{e}},
$$

and the photoheating/cooling time

$$
t_{h c}=0.1 \frac{e_{g a s}}{\dot{e}_{h t / c o o l}} .
$$

Their relative magnitudes can seamlessly evolve throughout a single application. For example, when an I-front propagates through a medium, photoheating times are often smaller than Courant times, and chemical time scales are usually shorter than either one. On the other hand, fossil H II regions can cool faster than they recombine, so cooling times become shorter than chemical times. The key to solving all three processes selfconsistently is to evolve each on its own timescale without restricting the entire algorithm to the shortest of the times. To successfully deal with both I-fronts and relic $H$ II regions, an algorithm must adaptively reshuffle the time scales on which the three processes are solved. Implicit schemes are sometimes applied to stiff sets of differential equations like those in our model because they are unconditionally stable over the Courant time. However, accurate I-front transport in stratified media often requires restricting updates to both the gas energy and fluid equations to photoheating times in order to capture the correct energy deposition into the gas, and linear systems solves over such short time scales would be prohibitive in more than one or two dimensions. Enforcing photon conservation in implicit schemes can also be problematic.

We instead subcycle chemical species and gas energy updates over the minimum of the chemical and heating/cooling times until the larger of the two has been crossed, at which point we perform full hydrodynamical 

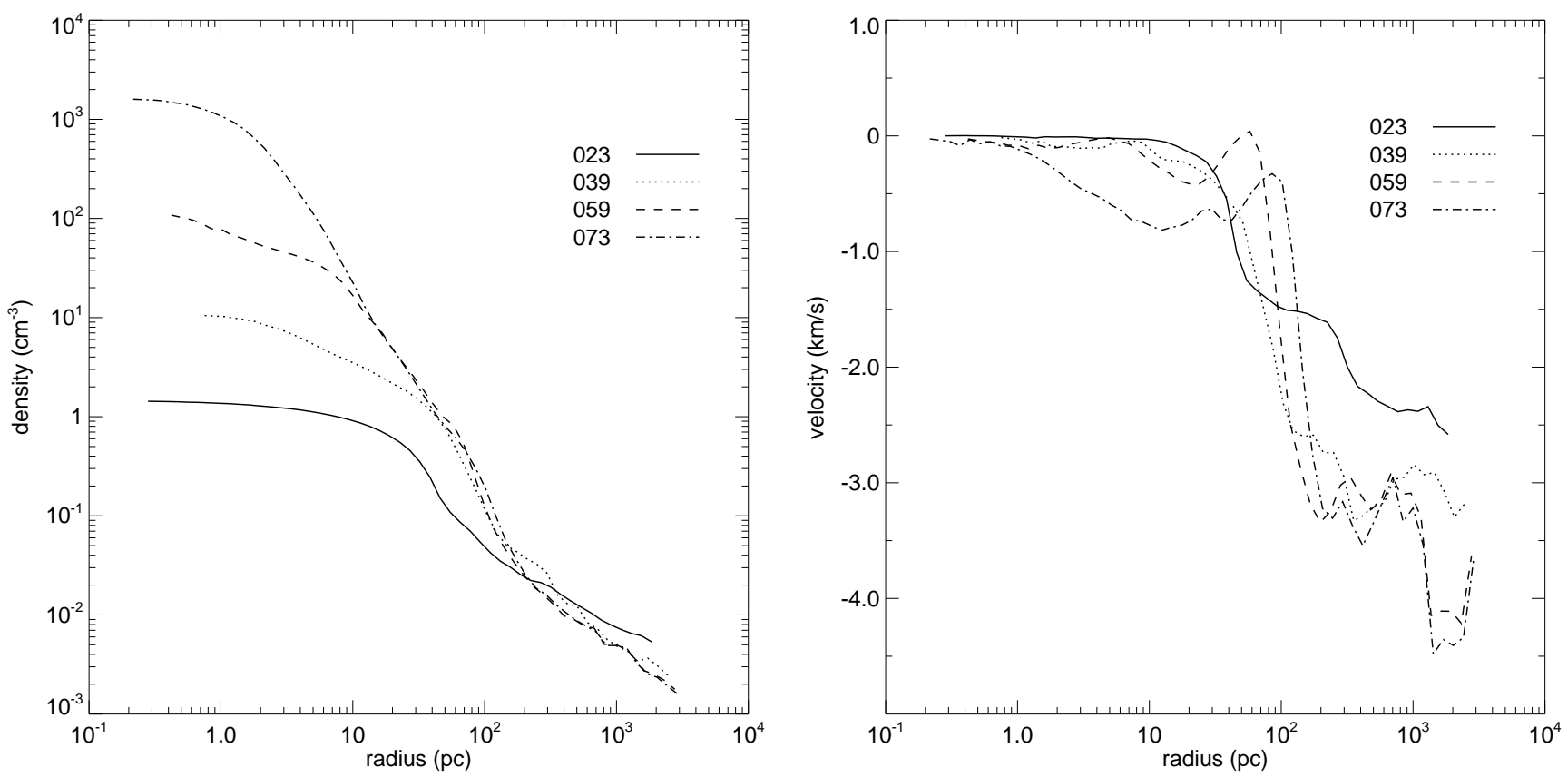

FIG. 1.- Spherically-averaged baryon profiles for the $1.35 \times 10^{5} \mathrm{M}_{\odot}$ halo at four stages of evolution. The redshifts of the 023, 039, 059, and 073 profiles are 23.9, 17.7, 15.6, and 15.0, respectively, with corresponding central densities of $1.43,10.5,108$, and $1596 \mathrm{~cm}^{-3}$. Left: densities. Right: radial velocities.

updates of gas densities, energies, and velocities. These times are global minima for the entire grid. The chemical times are defined in terms of electron flow to accommodate all chemical processes rather than just ionizations or recombinations. Adopting the minimum of the two times for chemistry and gas energy updates enforces accuracy in the reaction network when $t_{\text {chem }}$ becomes greater than $t_{h c}$, such as in relic H II regions. Our adaptive subcycling scheme is described in greater detail in (Whalen \& Norman 2008b).

\section{HALO MODELS}

The $1.35 \times 10^{5} \mathrm{M}_{\odot}$ halos we study are computed from cosmological initial conditions in the Enzo adaptive mesh refinement (AMR) code as described in detail in Whalen et al. (2008a). The halo baryon density and temperature profiles were spherically averaged and then mapped onto a two-dimensional cylindrical coordinate (ZR) grid in ZEUS-MP. The assumption of radial symmetry in two dimensions is approximate but valid, given the spheroidal nature of the halos. A single, symmetrized baryon profile better represents all halos of this mass than the three-dimensional profile of just this halo because small differences in angular distribution of the baryons are averaged out. We center the halo on the $z$-axis so that only its upper hemisphere resides on the grid. The mesh boundaries are $-125 \mathrm{pc}$ and $125 \mathrm{pc}$ in $z$ and $0.01 \mathrm{pc}$ and $125 \mathrm{pc}$ in $r$. The grid is 1000 zones in $z$ and 500 zones in $r$ with a spatial resolution of 0.25 pc. Outflow conditions are assigned to the upper and lower boundaries in $z$ and reflecting and outflow conditions are imposed on the inner and outer boundaries in $r$, respectively.

Dark matter gravity is included by constructing a separate potential that cancels pressure forces everywhere on the mesh, thus imposing hydrostatic equilibrium on the halo, and then holding the potential fixed throughout the simulation. Force updates to gas velocities are performed with the potential every hydrodynamical time step during the simulation. Neglecting dark matter dynamics introduces no serious errors because the gas in the halo evolves on much shorter time scales than either the Hubble time or merger times, which at $z \sim 20$ are approximately $20 \mathrm{Myr}$. Updates to the self-gravity of the gas are also performed every hydrodynamical time step by evolving Poisson's equation with a two-dimensional conjugate gradient (CG) solver.

In reality, the halo does have infall velocities as shown in Fig 1. but they are minor. Their inclusion would only slightly enhance core densities during photoevaporation. The composition of the gas in our models was primordial, $76 \% \mathrm{H}$ and $24 \% \mathrm{He}$ by mass. We assume ionized and $\mathrm{H}_{2}$ fractions of $1.0 \times 10^{-4}$ and $2 \times 10^{-6}$, respectively. These values are consistent with remnant free electron fractions from the era of recombination at the redshift $z=20$ we take for our models and with those expected from collisional ionizations in cosmological accretion shocks. We consider the four evolutionary stages of the halo in our earlier work, corresponding to central densities $n_{c}=$ $1.43,10.5,108$, and $1596 \mathrm{~cm}^{-3}$, respectively. Each halo profile is illuminated at 150, 250, 500, and $1000 \mathrm{pc}$, which are typical of interhalo separations in a cluster. Each profile is illuminated by $25,40,60$ and $80 \mathrm{M}_{\odot}$ stars for their respective lifetimes, 6.46, 3.86, 3.46 and 3.01 Myr, for a total of 64 models. These stars sample the lower end of the Population III mass spectrum. After the death of the star the halo is further evolved to a total of $10 \mathrm{Myr}$, or about half of a merger time, to determine the effect of relic $\mathrm{H}$ II region dynamics on the gas in the core. 


\subsection{Criteria for Star Formation}

Unlike AMR or Lagrangian SPH algorithms, the fixed Eulerian grid in ZEUS-MP lacks the resolution to follow the collapse of the baryons in the halo into a new star. However, numerical simulations demonstrate that primordial star formation is a very robust process: if a halo is in a state in which its baryons can form a star, it will within a few Myr. Since our halo is one in which star formation has already been proven to occur, if the final density and $\mathrm{H}_{2}$ fraction of its core is greater than or equal to its pre-illumination levels, a star will form at its center. In some cases the relic I-front shock passes through the center of the halo and imparts an impulse to the gas there. However, if the final velocity of the perturbed baryons within a parsec of the core is less than the escape speed from the halo, they will be trapped by the dark matter potential and still form a star. We therefore adopt the simple criteria that if central baryon densities and $\mathrm{H}_{2}$ fractions in the halo are the same or greater than those just before illumination, and if central gas velocities are lower than the escape speed, a star will form in the halo. If we approximate the binding energy $E_{\mathrm{B}}$ of the baryons to the halo by that of a homogeneous sphere,

$$
E_{\mathrm{B}}=\frac{3}{5} \frac{G M_{\mathrm{h}} m_{\mathrm{b}}}{R_{\mathrm{vir}}}
$$

where $M_{\mathrm{h}}, m_{\mathrm{b}}$, and $R_{\mathrm{vir}}$ are the total halo mass, baryon mass, and virial radius of the halo, respectively, then the escape speed $v_{\text {esc }}$ is

$$
v_{\mathrm{esc}} \sim\left(\frac{6}{5} \frac{G M_{\mathrm{h}} m_{\mathrm{b}}}{R_{\mathrm{vir}}}\right)^{\frac{1}{2}} .
$$

For the halo in our models, $v_{\text {esc }} \sim 2.6 \mathrm{~km} \mathrm{~s}^{-1}$.

\section{RESULTS}

The (Whalen et al. 2008a) study found several possible final fates for halos photoevaporated by a $120 \mathrm{M}_{\odot}$ star. If the halo is very diffuse, with $n_{c}<1 \mathrm{~cm}^{-3}$, it is completely evaporated anywhere it resides in the cluster, with no star formation. If the halo is more evolved, with $n_{c}>1000 \mathrm{~cm}^{-3}$, its core is shielded from both ionizing and LW flux and star formation proceeds there without delay. In halos of medium central density the relic ionization front shock either compresses and enriches the core with $\mathrm{H}_{2}$, potentially hastening its cooling and collapse into a star, or its residual momentum disrupts the core, delaying or suppressing star formation. For comparison to our $25-80 \mathrm{M}_{\odot}$ models, which are evolved to $10 \mathrm{Myr}$, we ran the $120 \mathrm{M}_{\odot}$ models of (Whalen et al. 2008a) out to the same time. We list final outcomes for star formation in these simulations according to the criteria set forth in section 3.1 in Figure 2.

\subsection{5 - $80 M_{\odot}$ Stars}

In Figures 3 and 4 we tabulate the effects of local ionizing and LW radiation on star formation in the halo near 25, 40, 60 and $80 \mathrm{M}_{\odot}$ stars. First, we note that in each of these stars new star formation obeys the same trends as in a $120 \mathrm{M}_{\odot}$ star: it is first suppressed, then delayed, and finally unaffected as its central baryon density and distance to the star grow. These trends are primarily due to

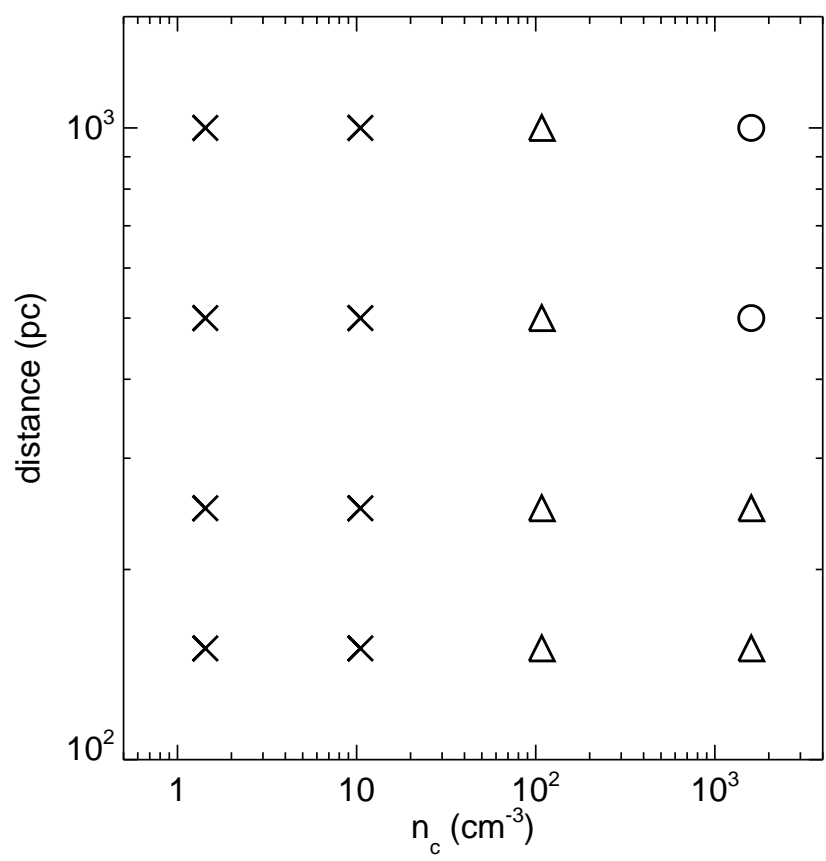

FIG. 2.- Star formation in a $1.35 \times 10^{5} \mathrm{M}_{\odot}$ halo in the vicinity of a $120 \mathrm{M}_{\odot}$ star, from Whalen et al. (2008a). The central baryon densities $n_{c}$ of the halo at the time of illumination were $1.43,10.5,108$, and $1596 \mathrm{~cm}^{-1}$, respectively. The $120 \mathrm{M}_{\odot}$ star was at 150, 250, 500 and 1000 pc. Completely evaporated halos with no star formation are labeled by crosses and halos with delayed or undisturbed star formation are represented by triangles and circles, respectively.

the momentum with which the I-front shock reaches the core of the halo. As explained earlier, when star formation is delayed it is due either to disruption of the center of the halo by the relic I-front shock, LW photodissociation of the core of the halo, or both. If star formation is unaffected it is because the core is completely shielded from LW flux, which also guarantees that the relic I-front shock will not reach the core before it forms a star. From the upper left to lower right in the 60 and $80 \mathrm{M}_{\odot}$ panels runs a line that marks the boundary between quenched star formation and delayed or undisturbed star formation (corresponding lines for 25 and $40 \mathrm{M}_{\odot}$ stars, which die in SN explosions whose kinetic feedback must also be taken into account, are shown in section 4.2). The suppression of new star formation in the models lying just below this line is usually unambiguous: the shock completely sweeps baryons from the core of the halo. Only in models lying just above this line does star formation vary with the mass of the illuminating star. The line advances gradually up and to the right with neighbor star mass, but the shift is minor from $25-80 \mathrm{M}_{\odot}$. In a halo at a fixed central baryon density and distance from the star, the formation of a new star is at most delayed if it was once unaffected, or halted if it was once delayed, as the mass of the illuminating star varies from $25-80 \mathrm{M}_{\odot}$. Star formation remains unchanged above and below this narrow band. We note that the lines are drawn to cut the space between symbols evenly and as such should be taken as order of magnitude estimates.

Why is star formation in the satellite halo relatively 

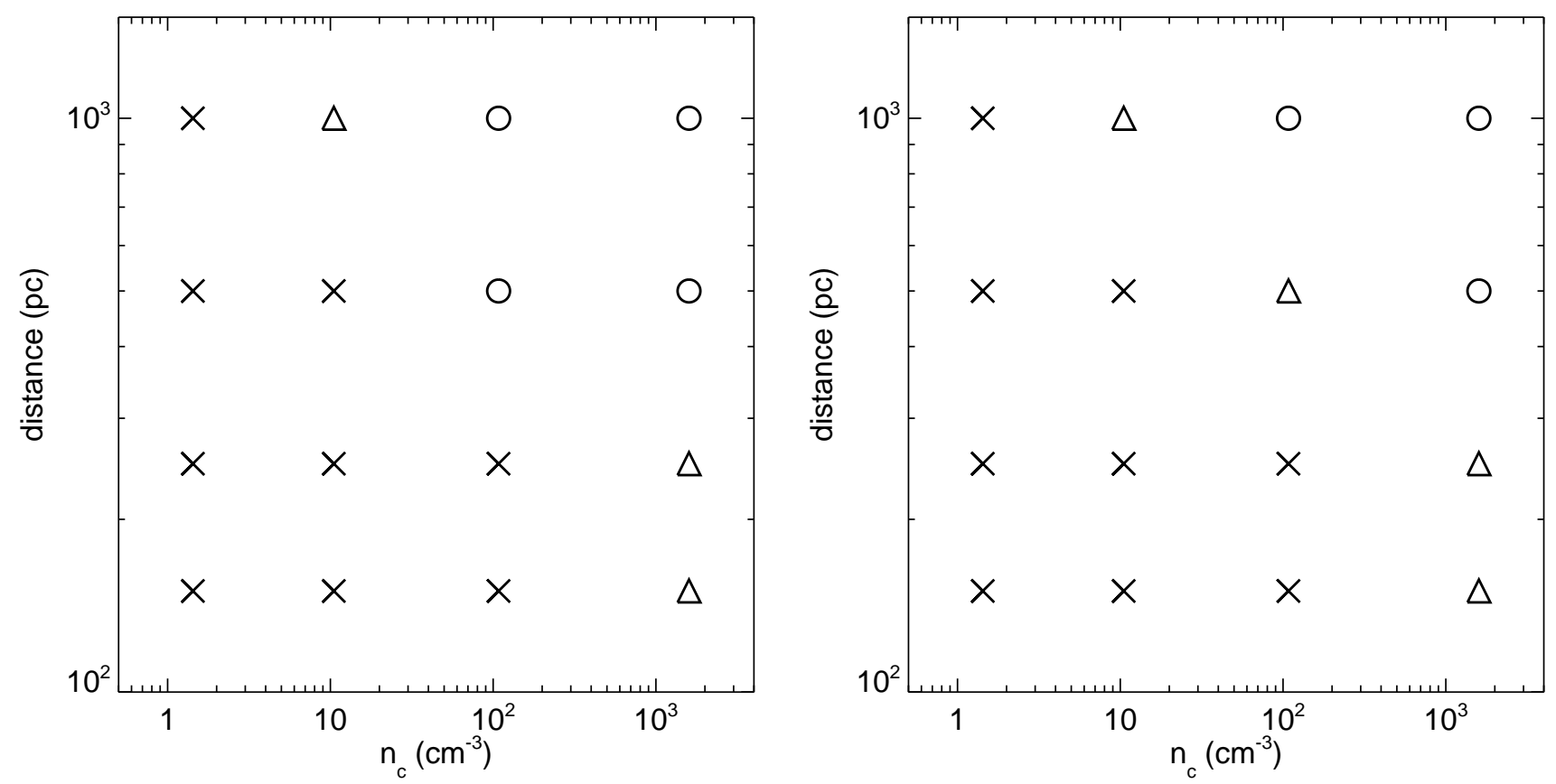

FIG. 3. - Star formation in the halo when illuminated by a $25 \mathrm{M}_{\odot}$ star (left) and a $40 \mathrm{M}_{\odot}$ star (right) at four central baryon densities $n_{c}, 1.43,10.5,108$, and $1596 \mathrm{~cm}^{-3}$, and at four distances from the star, 150, 250, 500 and 1000 pc, which are typical interhalo spacings within a cluster. Completely evaporated halos with no star formation are labeled by crosses, and halos with delayed or undisturbed star formation are indicated by triangles and circles, respectively.
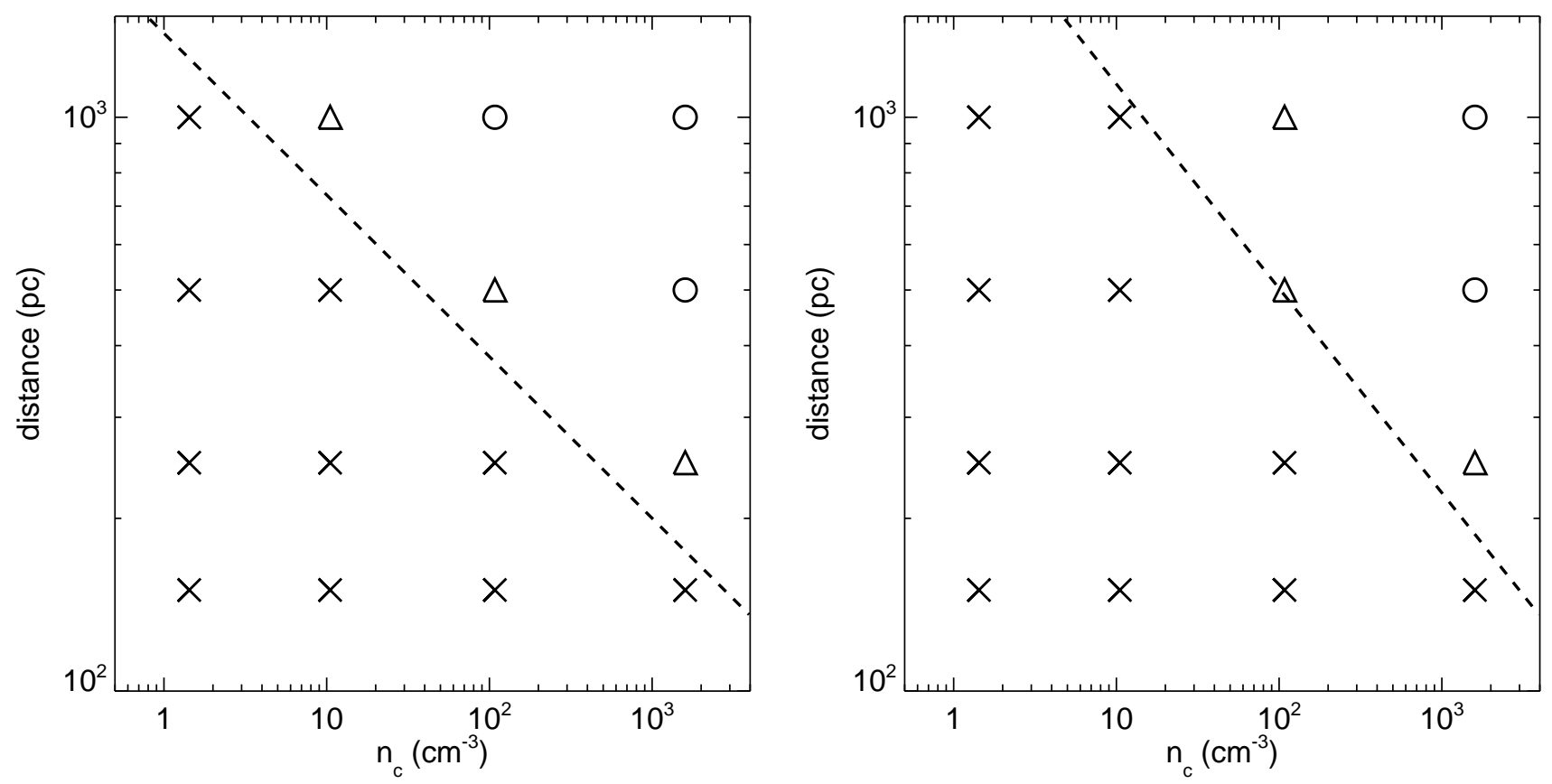

FIG. 4.- Star formation in the halo in the vicinity of a $60 \mathrm{M}_{\odot}$ star (left) and an $80 \mathrm{M}_{\odot}$ star (right) at the central gas densities and distances to the star considered in our study. Completely ionized halos with no star formation are labeled by crosses, and halos with delayed or undisturbed star formation are indicated by triangles and circles, respectively. The dotted lines mark the threshold for star formation in the evaporated halos, above which it proceeds and below which it is quenched. 
uniform with neighbor star mass? On one hand, the dimmer flux of low-mass Pop III stars causes the front to transform from R-type to D-type further out from the center of the halo. We plot velocity profiles for the Ifronts at the moment they become D-type in the $n_{c}=108$ $\mathrm{cm}^{-3}$ halo $500 \mathrm{pc}$ from $25-120 \mathrm{M}_{\odot}$ stars in panel (a) of Figure 5. In each profile the transition distance from the core is marked by the position of the forward peak, which is at $80,75,60,55$ and $50 \mathrm{pc}$ for the $25,40,60,80$ and 120 $\mathrm{M}_{\odot}$ stars, respectively. On the other hand, lower-mass stars are also longer lived, so they drive the I-front into the halo for greater times. This collapses the dispersion in I-front positions in the halo to $10 \mathrm{pc}$ by the time the star dies, as shown in panel (b) of Figure 5 In this halo, the I-fronts reach the core with nearly the same velocity, which is well below the escape speed, with each star. This pattern holds for all halos in which the I-front falls short of the core when the star dies, when star formation is delayed or uninterrupted. Note that at early times the velocity profile is split into two smaller peaks. This is due to penetration of hard UV photons into the dense shocked gas ahead of the front, which drives a backflow in the frame of the shock. This is a common feature of I-fronts due to hard UV spectra, as discussed in detail in section 4.1 and Figure 17 of Iliev et al. (2009). The evolution in spectral profile from $25-120 \mathrm{M}_{\odot}$, which causes ionized gas temperatures to rise by more than $50 \%$ in the H II region, accounts for the variation in peak velocity in Figure 5. Had each of these I-fronts been driven by a monochromatic flux of the same magnitude and duration, the spread in the peaks in radius would have been even less than $10 \mathrm{pc}$.

In the Whalen et al. (2008a) survey, the momentum imparted by the relic I-front shock to the core of the halo is primarily what determines if a new star forms there. Here, in a few cases, the compresssion of the shadow of the halo toward the axis also determines if a star forms. In our new models the halo forms a shadow on the same time scale as a $120 \mathrm{M}_{\odot}$ star but pressure from the surrounding relic $\mathrm{H}$ II region drives it inward toward the axis for up to twice the time before the star dies, squeezing a flow backward into the center of the halo. This backflow can slightly displace baryons from the core, as we show in Figure 6f for halo 073150 pc from a $60 \mathrm{M}_{\odot}$ star. The clump of gas originally centered in the dark matter potential retains its shape and is at well below the escape speed of the halo. However, its slight dislocation from the center of the dark matter potential at $10 \mathrm{Myr}$ prevents it from collapsing into the star that would have formed if only the relic I-front shock had been present. Backflows are especially collimated in the axial symmetry we assume in our models but we still expect them to be present in three dimensional halos because of their approximate sphericity.

In halos where stars form without interruption during photoevaporation, molecular hydrogen mass fractions rapidly rise from the initialized value of $2 \times 10^{-6}$ to $10^{-4}$ when the star is turned on, even with LW flux, because the core self-shields, as we show for the halo at $n_{c}=1596$ $\mathrm{cm}^{-3} 500 \mathrm{pc}$ from $25,40,60$, and $80 \mathrm{M}_{\odot}$ stars in the right panel of Figure 7 The core collapses even as the outer layers of the halo are stripped away by supersonic outflows, and a star forms just as quickly as in the absence of radiation. Since we begin with cosmic mean $\mathrm{H}_{2}$ fractions of $2 \times 10^{-6}$ instead of more realistic values of $10^{-4}$ for simplicity, ours are lower limits to self-shielding and cooling.

One departure of the $120 \mathrm{M}_{\odot}$ models from the others is the delayed star formation at 150 and $250 \mathrm{pc}$ in the halo at $n_{c}=108 \mathrm{~cm}^{-3}$, which does not occur near 25 $80 \mathrm{M}_{\odot}$ stars. This happens because the shock remnant traverses the core of the halo sooner, allowing gas to later pool in the dark matter potential and reach its original density at the center by $10 \mathrm{Myr}$, as shown by the dashed line in the left panel of Figure 7 at 150 pc. Such backfill allows a new star to form before a total of $20 \mathrm{Myr}$ has elapsed, or about a merger time at $z \sim 20$. Similar flows would occur in low-mass runs evolved beyond $10 \mathrm{Myr}$ but would probably not result in a new star prior to severe disruption by a merger. The higher densities at earlier times in the outer regions of the halo (solid lines in Figure 7) are due to compression by the relic I-front shock as it envelopes the halo.

We find that the higher ratio of LW to ionizing photons of low-mass Population III stars has no effect on nearby star formation. The lower fluxes of these stars offset their higher ratios, and in any event LW photons from a single star cannot prevent a new star from forming in a nearby halo prior to the appearance of LW backgrounds at lower redshifts. They can only delay it for the life of the proximate star. Without LW photons halo collapse times at $n_{c} \sim 2000 \mathrm{~cm}^{-3}$ are $7-10 \mathrm{Myr}$. At such densities, Whalen et al. (2008a) find that the core begins to strongly self-shield from local LW flux. If the core could collapse before the arrival of the I-front, its $n_{c}$ would be far greater, be fully shielded from LW flux, and form a star anyway. Halos that are completely ionized are too diffuse to form a star before the I-front reaches the core even in the absence of LW photons. Cores that form a star after the death of a nearby star could not have created one sooner without LW flux because their star formation times are even greater than $7 \mathrm{Myr}$. Finally, halos that are too severely disrupted by the relic I-front shock to form a star cannot collapse prior to the arrival of the shock in the absence of LW radiation for the same reason. Thus, in none of these outcomes would a star have formed if there had been no LW flux. In the latter two cases, we find that $\mathrm{H}_{2}$ fractions at the center of the halo reacquire their original values $100-200 \mathrm{kyr}$ after the star dies and that cooling and collapse of the core begins anew, augmented in some cases by molecular hydrogen advected into it by the relic I-front shock. Any suppression or delay of star formation is entirely due to bulk flows driven into the core driven by relic Ifront shock and shadow dynamics, not to the destruction of $\mathrm{H}_{2}$, which quickly reconstitutes in the core after the star dies. Thus, local ionizing UV flux governs new star formation in clustered halos, not local LW photons.

An exception to this is when the baryons are very close to the star, 25 - 150 pc. In such cases, when a star is irradiating a clump of baryons in the same halo, Hasegawa et al. (2009) find that clouds with freefall times that are shorter than ionization times can be prevented from collapsing by $25 \mathrm{M}_{\odot}$ stars because they have the highest LW/ionizing photon ratio. This never occurs in our models because of the much lower LW fluxes at typical halo distances within the cluster, but such scenarios are quite relevant to the formation of Population 

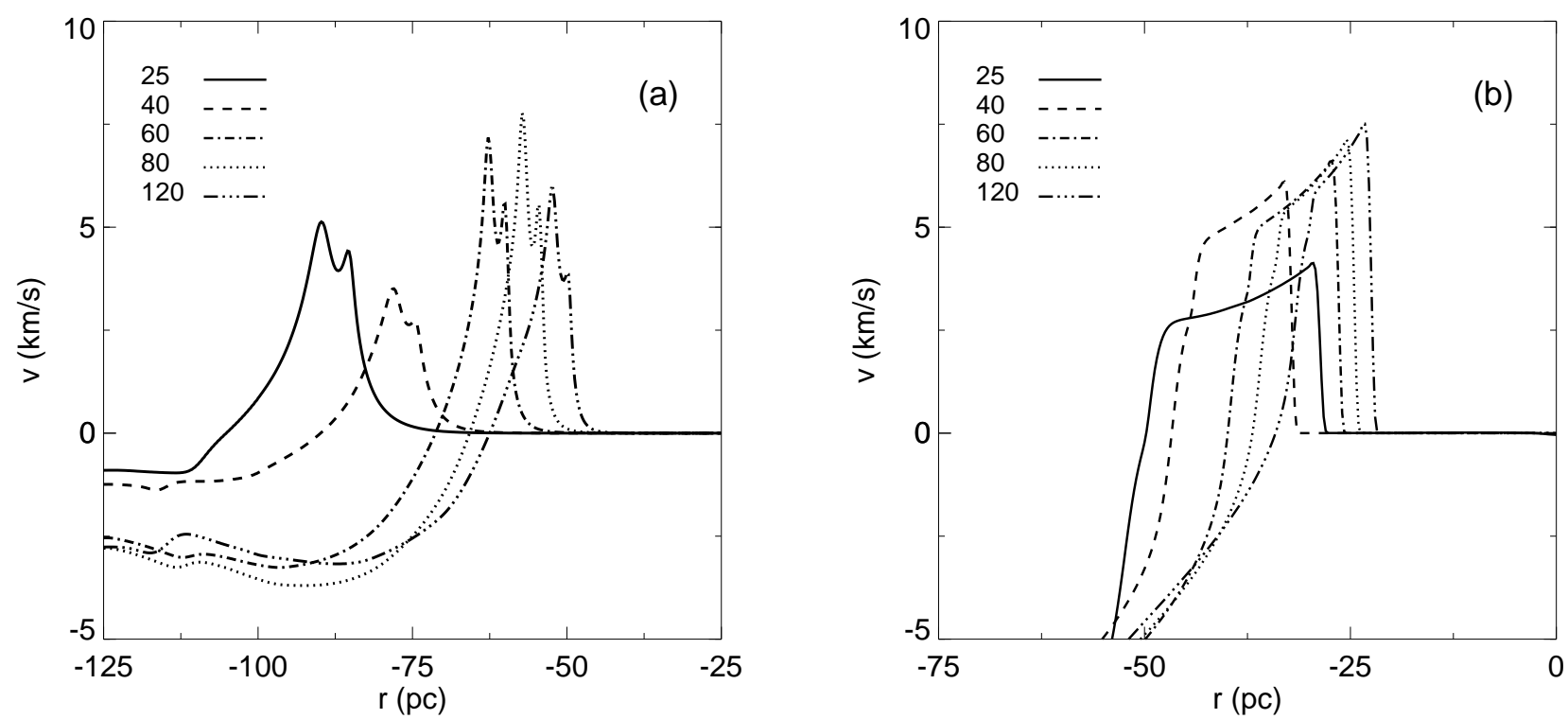

FIG. 5.- I-front gas velocity profiles through the central axis of the halo for $25,40,60,80$, and $120 \mathrm{M}_{\odot}$ stars $500 \mathrm{pc}$ from halo $059\left(n_{c}=\right.$ $108 \mathrm{~cm}^{-3}$ ). Panel (a): velocity at the time each front transforms from R-type to D-type. Panel (b): gas velocity profiles at the time each star dies.

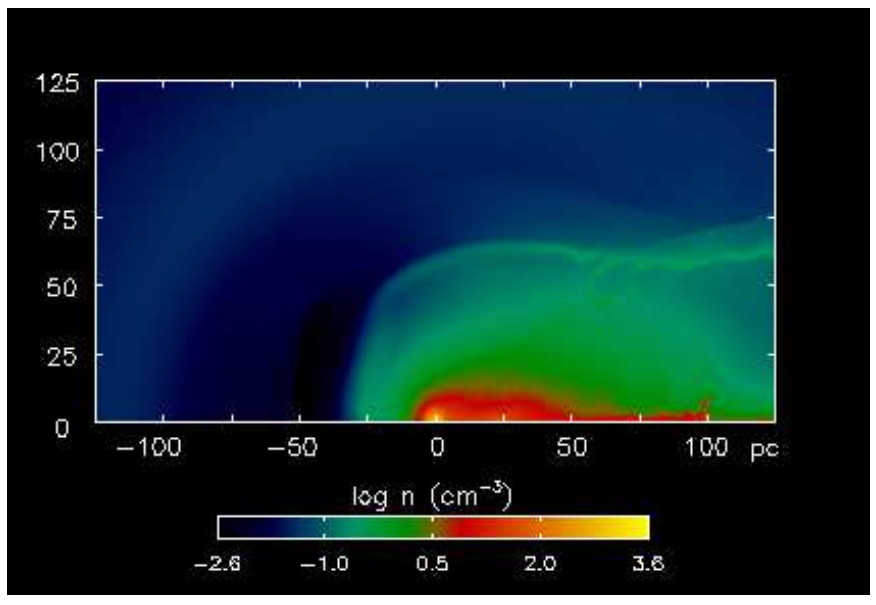

Fig. 6.- Evaporated halo with $n_{c}=1596 \mathrm{~cm}^{-3}$ (halo 073) 150 pc from a $60 \mathrm{M}_{\odot}$ star at $10 \mathrm{Myr}$. The core of the halo is slightly displaced to the left of center by backflow from the collapsed shadow on the right.

III binaries within a halo (Turk et al. 2009). Persistent LW backgrounds at lower redshifts can by themselves prevent secondary star formation in the cluster by not allowing partially evaporated cores to cool after the death of the star. However, the exclusion of radiation hydrodynamical effects in past studies may have led them to overestimate this effect, as we discuss in $\S 5$.

\subsection{Kinetic Feedback by $\mathrm{SNe}$}

Unlike the $100-120 \mathrm{M}_{\odot}$ stars assumed in most local radiative feedback studies, $25-50 \mathrm{M}_{\odot}$ stars die in SN explosions (Heger \& Wooslev 2002; Tominaga et al. 2007). Their remnants can ram-pressure strip baryons from nearby halos and prevent them from forming a star when one otherwise would have been created. Sakuma \& Susa (2009) examined kinetic feedback by SNe on star formation in satellite halos with semi-analytical arguments that neglected radiative preprocessing of the halo by the progenitor star. They found that SNe generally preempt any star formation that fails to occur prior to the arrival of the remnant at the halo. Cen \& Riquelme (2008) simulated the interaction of SN remnants with much more massive halos at lower redshifts to assess the degree to which metals become mixed with gas deep in their interiors. These calculations, which also excluded prior photoevaporation by UV backgrounds, found some mixing in the outer layers of the halos due to Kelvin-Helmholtz instabilities but that no metals reached their interiors. These models did not address SNe feedback on star formation because the potential wells of the halos were much deeper than those in which the first stars formed, so the remnants could not strip gas from their cores. Detailed numerical models of the collision of primordial supernova remnants with the relic H II regions enveloping partially exposed cores are needed to establish the actual fate of star formation in these circumstances.

Supernovae are not invariably fatal to local star formation because the time scales on which the shock reaches outlying halos may be longer than those on which baryons collapse within them, and in some cases the remnant never reaches them. Greif et al. (2007) found that SN remnants typically expand to only half the radius of the relic H II region of the progenitor because they come into pressure equilibrium relatively quickly in the warm recombining gas. Whalen et al. (2008b) computed the radius of the remnant as a function of time for core collapse SNe, hypernovae, and pair-instability SNe (PISN) in the fossil H II regions of $10^{5}-10^{7} \mathrm{M}_{\odot}$. In their simulations, ejecta from the explosions of $15-40 \mathrm{M}_{\odot}$ stars propagate at most $400-500 \mathrm{pc}$ in $10 \mathrm{Myr}$. We plot the radii 

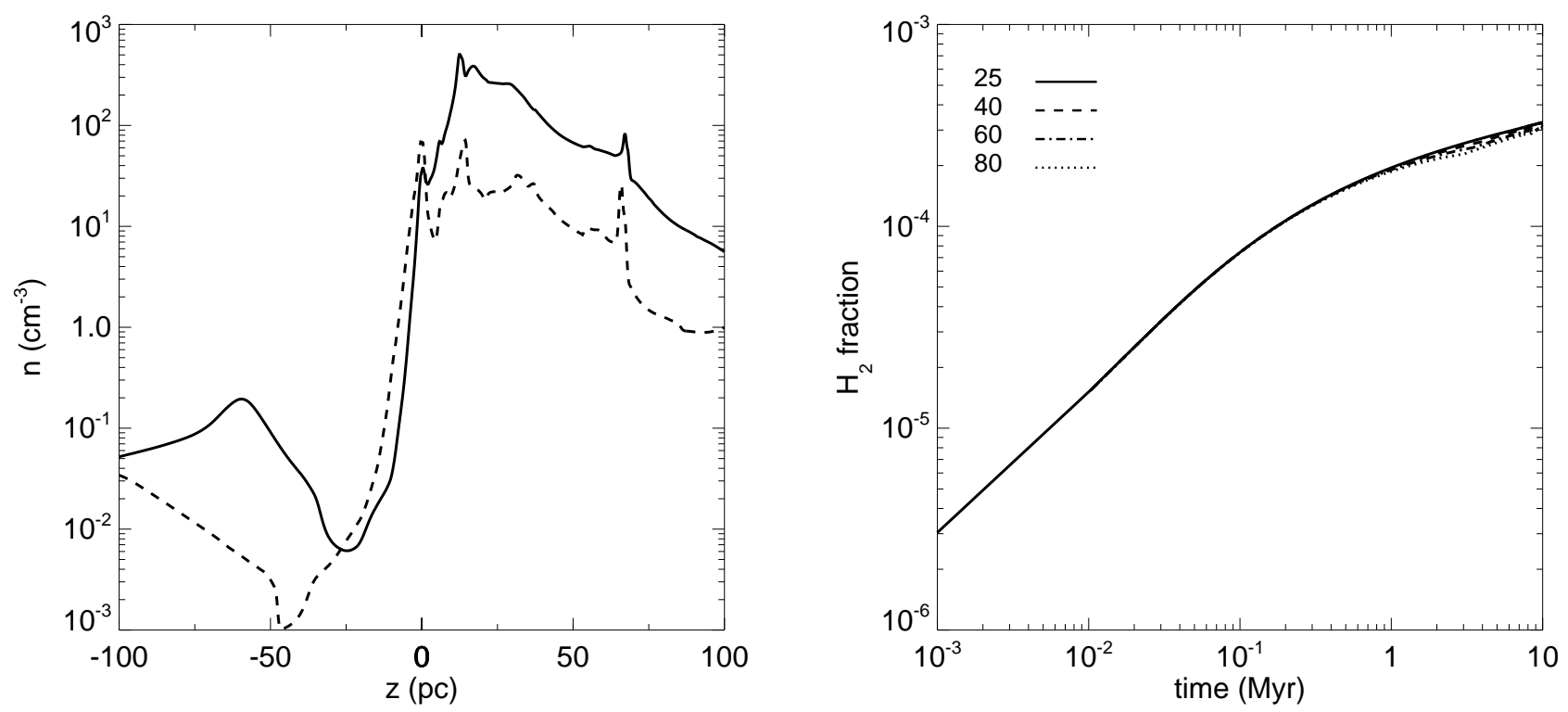

FIG. 7.- Left panel: the flow of baryons back into the center of the dark matter potential from the relic H II region, as shown in these density profiles along the central axis of the halo. Solid: 5.5 Myr; dashed: 10 Myr. Right panel: $\mathrm{H}_{2}$ mass fractions at the center of halo $073500 \mathrm{pc}$ from $25,40,60$ and $80 \mathrm{M}_{\odot}$ stars from zero to $10 \mathrm{Myr}$.

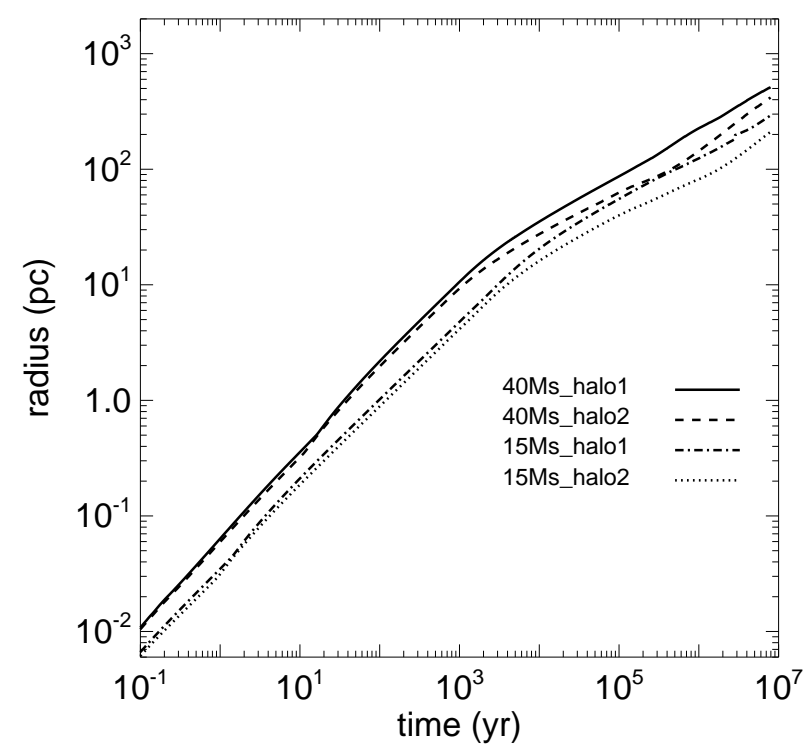

FIG. 8.- Radii of $15 \mathrm{M}_{\odot}$ supernova and $40 \mathrm{M}_{\odot}$ hypernova remnants in $6.9 \times 10^{5} \mathrm{M}_{\odot}$ and $2.1 \times 10^{6} \mathrm{M}_{\odot}$ halos (halo1 and halo2, respectively) from Whalen et al. (2008b).

of 15 and $40 \mathrm{M}_{\odot} \mathrm{SN}$ remnants in those models in Figure 8. As shown in Figure 10 of Whalen et al. (2008b), the growth of these remnants does not reproduce the idealized broken power-law radii of canonical SNe, chiefly because they do not exhibit self-similar behavior. Multiple reverse shocks reverberate throughout the remnant over its evolution, and it later violently collides with the dense H II region shell formed by its progenitor (see also Bromm et al. 2003; Kitavama \& Yoshida 2005).

If we adopt the conservative stance that a SN remnant halts star formation in a halo as soon as it reaches it, and if the collapse time of the halo at the time of the explosion is known, Figure 3 can be modified to include both radiative and kinetic feedback by the star. This is done simply by assuming that if a star can form in the evaporated halo in the absence of an explosion, it will still form if central baryon collapse takes less time than required for the shock to reach the halo. The contraction of the cloud from $n_{c} \sim 10-10^{3}$ to $10^{8} \mathrm{~cm}^{-3}$ is leisurely, taking place over 5 - 10 Myr (e.g. Abel et al. 2002; O'Shea \& Norman 2007). Thereafter, as three-body $\mathrm{H}_{2}$ cooling takes over in the core of the halo, the formation of a star proceeds very rapidly, in under a Myr. We therefore take $8 \mathrm{Myr}$ as the average time for a partially evaporated halo to form a star. Clearly, this estimate is approximate because it neglects the initial LW dissociation of the core, but it is reasonable given that our studies show that $\mathrm{H}_{2}$ reforms there 100 - $200 \mathrm{kyr}$ after the death of the star.

Arrival times for the shock at the center of the halo are complicated by the fact that they are a function of both the energy of the explosion and the mass of its host halo, as shown in Figure 8. We tabulate minimum and maximum arrival times for the remnant at 150, 250 and $500 \mathrm{pc}$ in Table 1 using the $15 \mathrm{M}_{\odot} \mathrm{SN}$ and $40 \mathrm{M}_{\odot}$ hypernova from (Whalen et al. 2008b) as proxies for the 25 and $40 \mathrm{M}_{\odot}$ SNe in our study. The evolution of the 15 $\mathrm{M}_{\odot}$ remnant is a reasonable approximation to that of the $25 \mathrm{M}_{\odot}$ remnant, given the uncertainty in explosion energy in primordial stars over this mass range. The hypernova, whose explosive yield is ten times greater than that of a $40 \mathrm{M}_{\odot}$ core-collapse $\mathrm{SN}$, gives a lower limit to the arrival times of the remnant to the halo. The dispersion in times with host halo mass is due to the extra baryons the remnant must sweep aside to reach the halo, which can vary by more than a factor of ten in mass. The host halos we have chosen, $6.9 \times 10^{5} \mathrm{M}_{\odot}$ and $2.1 \times$ $10^{6} \mathrm{M}_{\odot}$, bracket those in which the remnant actually es- 
TABLE 1

SN Remnant Propagation Times

\begin{tabular}{rrrrr}
\hline \hline $\mathrm{SN}$ & halo $\left(M_{\odot}\right)$ & $t_{150 p c}(\mathrm{yr})$ & $t_{250 p c}(\mathrm{yr})$ & \multicolumn{1}{c}{$t_{500 p c}(\mathrm{yr})$} \\
\hline $15 \mathrm{M}_{\odot}$ & $6.9 \mathrm{E}+05$ & $1.3 \mathrm{E}+06$ & $5.5 \mathrm{E}+06$ & $>1.0 \mathrm{E}+07$ \\
$15 \mathrm{M}_{\odot}$ & $2.1 \mathrm{E}+06$ & $3.5 \mathrm{E}+06$ & $>1.0 \mathrm{E}+07$ & $>1.0 \mathrm{E}+07$ \\
$40 \mathrm{M}_{\odot}$ & $6.9 \mathrm{E}+05$ & $3.1 \mathrm{E}+05$ & $1.1 \mathrm{E}+06$ & $8.0 \mathrm{E}+06$ \\
$40 \mathrm{M}_{\odot}$ & $2.1 \mathrm{E}+06$ & $8.5 \mathrm{E}+05$ & $2.4 \mathrm{E}+06$ & $>1.0 \mathrm{E}+07$
\end{tabular}

capes the halo since neither progenitor can ionize halos greater than $10^{7} \mathrm{M}_{\odot}$ (Whalen et al. 2008b). Since the hypernova remnant, which is the fastest, travels at most $500 \mathrm{pc}$ in $10 \mathrm{Myr}$, star formation at 500 and $1000 \mathrm{pc}$ in Figure 3 is unchanged.

We summarize both radiative and kinetic feedback by 25 and $40 \mathrm{M}_{\odot}$ stars in Figure 9. Ram pressure stripping at most preempts delayed star formation in the densest halo ionized by the $25 \mathrm{M}_{\odot}$ star at 150 and $250 \mathrm{pc}$; the less destructive of the $25 \mathrm{M}_{\odot}$ remnants only halts star formation at $150 \mathrm{pc}$. On the other hand, both $40 \mathrm{M}_{\odot}$ remnants shut down delayed star formation at $n_{c}=1596$ $\mathrm{cm}^{-3}$ at 150 and $250 \mathrm{pc}$. We find that kinetic feedback only modifies radiative feedback in halos at $400 \mathrm{pc}$ or less from low-mass stars. The picture is more complicated with much more energetic PISN, which can reach any halo within a typical cluster $(r \lesssim 1000 \mathrm{pc})$ in under 10 Myr (Figure 10 of Whalen et al. 2008b). Although the remnant overruns the halos sooner, radiative feedback can also speed up baryon collapse beforehand. Whether or not stars form in such cases remains unclear, and is beyond the scope of this study.

We note that our estimates of kinetic feedback are conservative for several reasons. First, as stated earlier, we apply arrival times for a $40 \mathrm{M}_{\odot}$ hypernova to both 25 and $40 \mathrm{M}_{\odot}$ core-collapse $\mathrm{SNe}$, which have lower energies and velocities, and hence a shorter reach within the cluster. Second, we assume that the halos in our study are not connected by filaments of dark matter and gas, which is usually not the case. If both the UV and the SN shock must propagate along a cosmological filament, with overdensities of 50 or more above the cosmic mean, to reach the core of a nearby halo they may have considerably less impact on subsequent star formation there. Finally, we also neglect the collision of the remnant with supersonic backflow from the evaporated halo, which will dampen its impact with the core of the halo. Also, $40-50 \mathrm{M}_{\odot}$ primordial stars may die in more exotic explosions such as hypernovae or collapsars that are either asymmetric or beamed (e.g. Tominaga et al. 2007; Tominaga 2009). However, the asphericity of such events lowers the likelihood that any one halo in a cluster would be struck by ejecta, so for simplicity we do not consider them here.

\subsection{Analytical Fits to Local Star Formation at High Redshift}

In our previous study we made the distinction between positive, negative, and neutral feedback within in a cluster of halos. Negative feedback meant that star formation is either delayed or suppressed, positive feedback meant that a star forms more quickly in the halo than when there is no radiation, and neutral feedback meant that a star forms as fast as it would without UV. However, what is ultimately of importance to early structure formation
TABLE 2

Analytical Fits to Radiative and Kinetic FeEdBack

\begin{tabular}{ccrr}
\hline \hline$M_{\star}$ & $a_{1}$ & \multicolumn{1}{c}{$b_{1}$} & \multicolumn{1}{c}{$c_{1}$} \\
\hline $25 \mathrm{M}_{\odot}$ & 3.3010 & -0.33474 & -0.14613 \\
$40 \mathrm{M}_{\odot}$ & 3.1761 & -0.49480 & 0.30103 \\
$60 \mathrm{M}_{\odot}$ & 3.3010 & -0.33011 & $9.6910 \mathrm{E}-02$ \\
$80 \mathrm{M}_{\odot}$ & 3.3010 & -0.33084 & -0.69897
\end{tabular}

and the rise of the first stellar populations is whether or not a star forms in the halo, not its exact timing, which varies by less than a few Myr in the scenarios we have investigated. Since the final result of neutral, positive, or no feedback is the formation of a star, the distinction between these outcomes is not relevant. Hence, the rules we formulate delineate the basic threshold for star formation in a halo exposed to UV flux and SN flows from nearby primordial stars.

In Figures 4 and 9 we plot lines that denote the threshold radius $r_{t h}$ from the star above which the halo can form a star if it is at a central gas density $n_{c}$ and below which it cannot. The threshold has the simple form

$$
\log _{10} r_{t h}=a_{1}+b_{1}\left(\log _{10} n_{c}+c_{1}\right) \text {, }
$$

where $r_{t h}$ is in pc and $n_{c}$ is in $\mathrm{cm}^{-3}$. We tabulate $a_{1}, b_{1}$ and $c_{1}$ in Table 2 for $25,40,60$ and $80 \mathrm{M}_{\odot}$ stars, including both radiative and kinetic feedback for 25 and $40 \mathrm{M}_{\odot}$ stars. As shown in Figure 2 radiative feedback by $120 \mathrm{M}_{\odot}$ stars is even simpler: if the satellite halo has central densities greater than $100 \mathrm{~cm}^{-3}$ a new star will form anywhere in the cluster. If not, its formation is suppressed.

\subsection{Ionization Front Instabilities}

Longer illumination times and lower fluxes promote the onset of dynamical instabilities in the D-type ionization front as it engulfs the satellite halo, as we show for the 073 halo $150 \mathrm{pc}$ away from a $25 \mathrm{M}_{\odot}$ primordial star in the left panel of Figure 10. They arise because the I-front assumes a cometary shape and because the high energy tail of the spectrum forms $\mathrm{H}_{2}$ between the front and the dense shell that radiatively cools the shell (e.g. Ricotti et al. 2001; Whalen \& Norman 2008a). Using rigorous perturbation analysis, Williams (2002) discovered that D-type fronts driven by photons that are oblique to the front are always unstable, and that the growth rates of the modes rise with the angle of incidence of the photons. This, together with cooling of the shocked shell by $\mathrm{H}_{2}$, is the origin of the instabilities in the I-front enveloping the halo in Figure 10.

At early times, we find that the modes with the greatest amplitudes are indeed those furthest out along the arc of the I-front, where photons are incident to the front at the greatest angles. At intermediate and later times the perturbations grow nonlinearly and degenerate into turbulent fluid motion along the outer segments of the arc. The amplitudes of the modes closest to the axis of the halo are small and instabilities never puncture its core. Much more prominent perturbations have been found in planar I-fronts approaching spherical molecular cloud cores in numerical models with efficient radiative cooling by molecules (Mizuta et al. 2006). These phenomena 

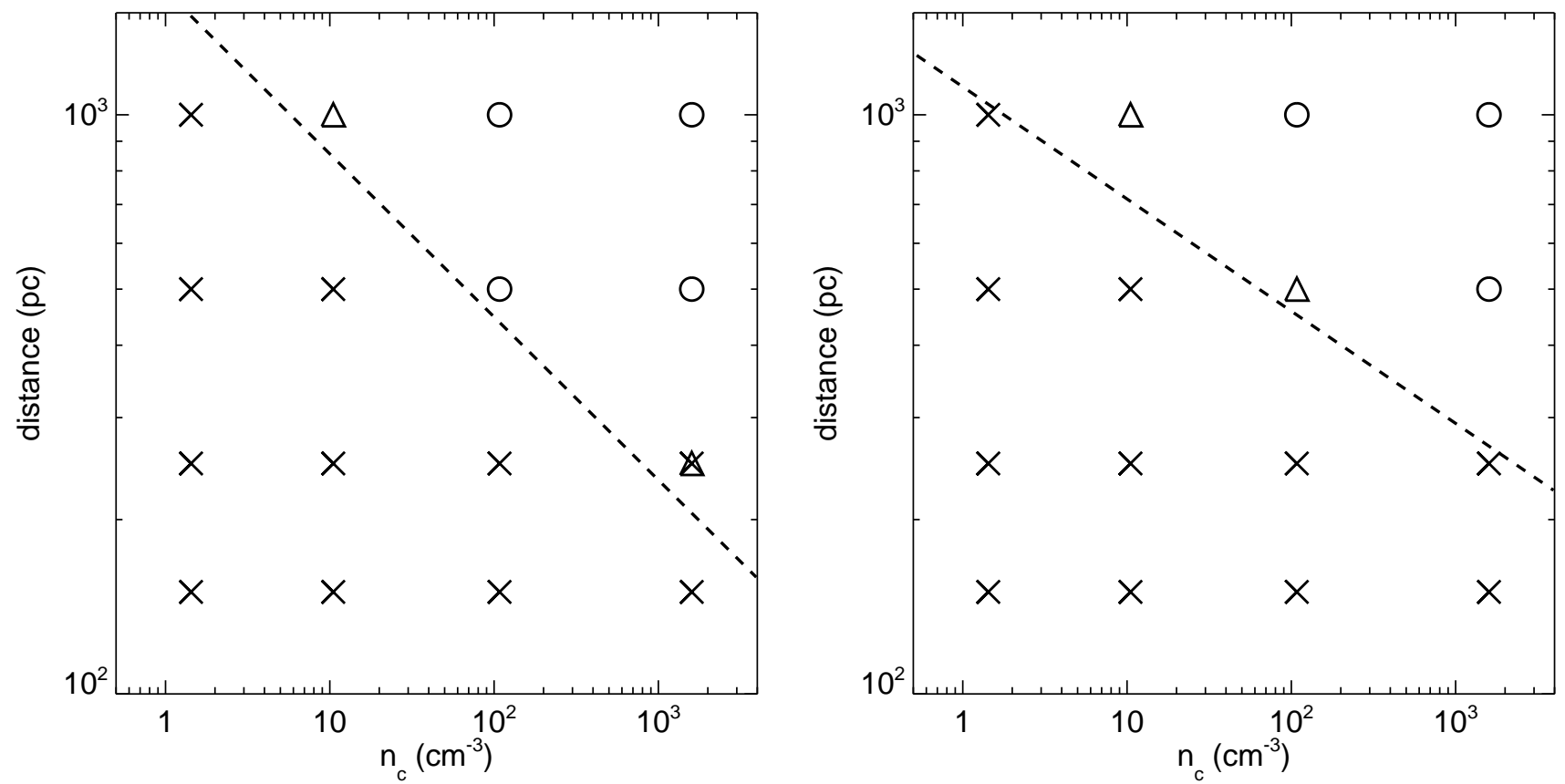

FIG. 9.- Radiative and kinetic feedback on star formation near a $25 \mathrm{M}_{\odot}$ star (left) and a $40 \mathrm{M}_{\odot}$ star (right). Completely evaporated halos with no star formation are labeled by crosses, while halos with delayed or neutral star formation are marked by triangles and circles, respectively. The triangle overlaid on the cross in the $25 \mathrm{M}_{\odot}$ panel signifies that the halo can form a delayed star if the SN goes off in the $6.9 \times 10^{5} \mathrm{M}_{\odot}$ halo but not if it is in the $2.1 \times 10^{6} \mathrm{M}_{\odot}$ halo. The dotted lines again define the boundary for star formation in the evaporated halos, above which it proceeds and below which it is quenched.

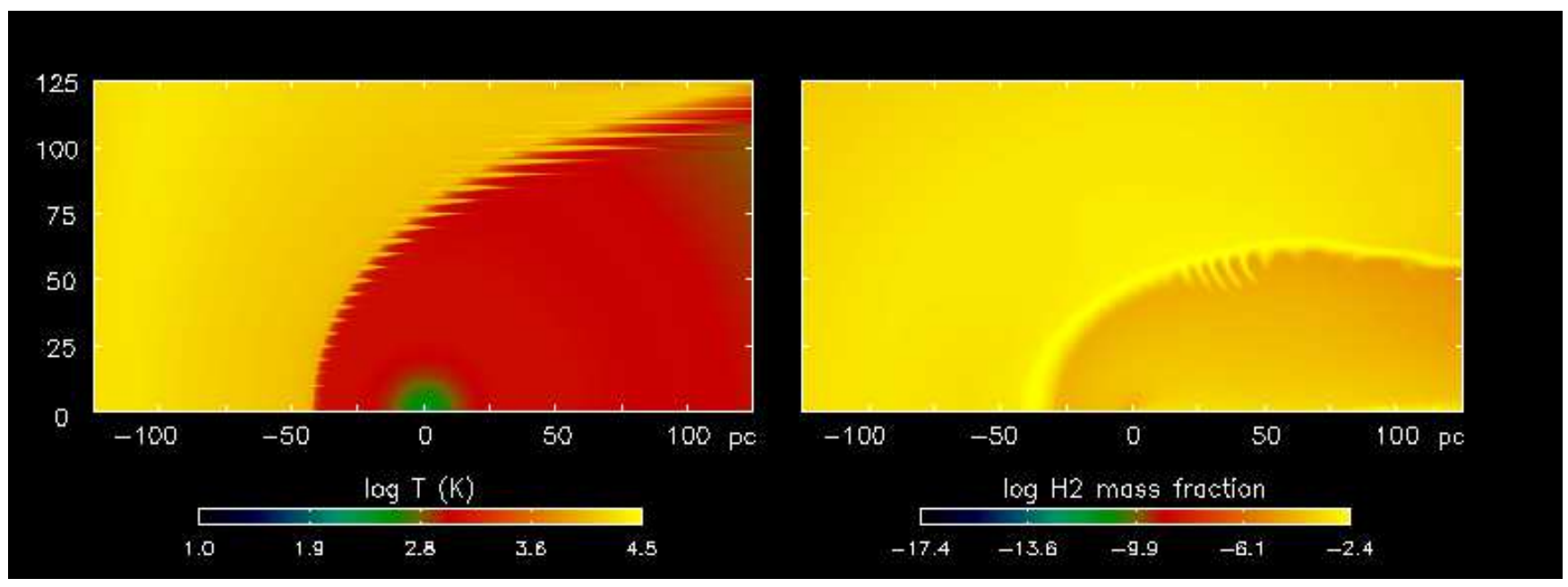

Fig. 10.- Temperatures at $600 \mathrm{kyr}$ (left) and $\mathrm{H}_{2}$ mass fractions at $10 \mathrm{Myr}$ (right) in halo $073\left(n_{c}=1596 \mathrm{~cm}^{-3}\right) 150 \mathrm{pc}$ from a $25 \mathrm{M} \odot$ star.

have been proposed for the origin of the "Pillars of Creation" in the Orion Nebula, but they are different from those in our simulations. They begin as Vishniac thinshell overstabilities (Vishniac 1983) caused by efficient molecular cooling in plane-parallel I-fronts, not curved ones, and later erupt into violent instabilities driven by ionizing radiation. In the Mizuta et al. (2006) models the unstable modes do propagate into the molecular cloud core. This never occurs in our simulations because $\mathrm{H}_{2}$ cooling is too inefficient to incite Vishniac modes.

We point out these features because they are promi- nent in many of our models, but they do not affect star formation in the halo because they never approach its inner regions. Mostly, they just roil gas along the shock, breaking it up into clumps that can persist for up to 10 Myr. We find that they appear when the star is 150 or $250 \mathrm{pc}$ from the halo and are most prominent with 25 and $40 \mathrm{M}_{\odot}$ stars. Fewer instabilities appear as stellar mass increases; they arise in only two of the $120 \mathrm{M}_{\odot}$ models and have lower amplitudes. There are two reasons for this. First, higher mass stars have greater LW fluxes that lower $\mathrm{H}_{2}$ cooling in the dense shell. Second, 
larger ionizing UV fluxes result in shorter-lived cometary profiles in which unstable modes can develop. The arc is crushed downward into the shadow of the halo more quickly and the instabilities have less time to develop.

\section{DISCUSSION AND CONCLUSION}

We find that $25-120 \mathrm{M}_{\odot}$ primordial stars are relatively uniform in their effect on new star formation within clusters of small halos at high redshifts, before the rise of global LW backgrounds. The evolution in spectral profile from $25-120 \mathrm{M}_{\odot}$ has no impact on the formation of stars in nearby halos, which allows its removal from the parameter space of local radiative feedback. The empirical fits we have devised mark the threshold for star formation in satellite halos as a function of central baryon density, proximity to the star, and neighbor star mass. Although the halo in our study is the just the least massive one found to form a star in previous AMR simulations, our results can be used as upper limits to feedback in more massive halos. Our results imply that future surveys of local feedback with more massive halos can be accomplished with fewer stars, since outcomes for halo photoevaporation above and below the belt in $n_{c}$ and radius in which there is variability is relatively uniform from $25-80 \mathrm{M}_{\odot}$.

Radiative and kinetic feedback between minihalos is key to many processes in early cosmological structure formation, such as primordial SNe event rates (Wise \& Abel 2005), especially those that account for cluster bias (MacIntyre et al. 2006), the rise of the first stellar populations, the assembly of primeval galaxies, and the evolution of metagalactic LW backgrounds. Our analytical fits enable feedback estimates for a representative cut of Population III stars in analytical models of these early processes. They can also be used in numerical simulations, especially those performed in large cosmological boxes capable of resolving minihalo clustering but not of capturing halo photoevaporation.

Local radiative feedback at slightly lower redshifts is different due to the presence of LW backgrounds from primordial stars, which until recently has been thought to be quite destructive to new star formation mediated by $\mathrm{H}_{2}$ cooling in halos (Haiman et al. 1997, 2000; Yoshida et al. 2003). However, recent, more detailed simulations reveal that star formation in cosmological halos is postponed rather than prevented in LW backgrounds, even large ones that are consistent with a fully reionized universe (Wise \& Abel 2007; O'Shea \& Norman 2008). Wise \& Abel (2007) found that a halo that formed a star at $5 \times 10^{5} \mathrm{M}_{\odot}$ in the absence of a photodissociative background still formed one by $\mathrm{H}_{2}$ cooling $50 \mathrm{Myr}$ later after it grew by mergers and accretion to $5 \times 10^{6} \mathrm{M}_{\odot}$ in a uniform LW field of $1 J_{21}\left(=10^{-21} \mathrm{erg} \mathrm{cm}^{-2} \mathrm{~Hz}^{-1} \mathrm{str}^{-1}\right.$ $\mathrm{s}^{-1}$ ), that of a fully-ionized universe. Other halos in its vicinity also grew to larger masses, even though cooling and collapse of baryons were temporarily stalled in them. Thus, at lower redshifts local UV feedback still begins when one star irradiates neighbor halos. In contrast to the first generation, LW backgrounds may continue to suppress star formation in photoevaporated halos after the death of the star by slowing the formation of $\mathrm{H}_{2}$ in the relic $\mathrm{H}$ II region, the remnant I-front shock, and the halo core.

However, this effect may have been overestimated in previous analyses. Consider the morphology and large densities and $\mathrm{H}_{2}$ fractions in the relic $\mathrm{H}$ II region at 10 Myr in the right panel of Figure 10. Similar $\mathrm{H}_{2}$ fractions would likely persist in the recombining $\mathrm{H}$ II region even in large LW backgrounds because the very high electron fractions there restore it so quickly via the $\mathrm{H}^{-}$channel. This is especially true in the high densities of the I-front shock remnant, that can be seen to envelope the core in Figure 10. Enough molecular hydrogen could be catalyzed in the envelope to expel the LW background from its interior, allowing $\mathrm{H}_{2}$ to reform at the center of the halo, cool it, and form a star. Thus, photoevaporation may actually free satellite halos to form stars that were previously suppressed by the background. This, together with our current study, suggests that star formation in cosmological halos at lower redshift was much more robust than is often supposed. Numerical models are now being developed to investigate the survival of molecular hydrogen, and therefore new star formation, in evaporated halos for a range $\mathrm{LW}$ backgrounds.

The effect of photoevaporation on the final mass of any star that does form in the halo is not yet well understood, but initial estimates by Susa et al. (2009) suggest that it will be smaller than in undisturbed halos. They find that outflows and shock disruption in the core lower central accretion rates, and by extrapolating these rates from early stages of collapse out to Kelvin-Helmholtz contraction time scales, they conclude that the final star will be $25-50 \mathrm{M}_{\odot}$. This mass scale is similar to those on which Pop III.2 stars form due to HD cooling in relic H II regions (Yoshida et al. 2007). HD is important because it can cool primordial H II regions down to the CMB temperature and lower the mass scales on which they fragment. However, we do not include it in our models because it forms primarily in the relic ionized gas surrounding the halo core, not in the core itself.

Although our study is a significant extension to our earlier survey of local radiative feedback, additional feedback channels remain to be properly investigated. If the death of the star results in a black hole, accretion would expose nearby halos to its soft $\mathrm{x}$-ray flux (Machacek et al. 2003; Alvarez et al. 2009), creating significant free electron fractions in them due to secondary ionizations without strongly heating them. This process could enhance their $\mathrm{H}_{2}$ mass fractions and promote their collapse into new stars. Likewise, the impact of SN ejecta with a halo that has been partially stripped by supersonic flows could deposit metals into its interior and accelerate its cooling and collapse. These potential avenues of positive feedback on primordial star formation will be the focus of future simulations.

This work was carried out under the auspices of the National Nuclear Security Administration of the U.S. Department of Energy at Los Alamos National Laboratory under Contract No. DE-AC52-06NA25396. The simulations were performed on the open cluster Coyote at Los Alamos National Laboratory. 


\section{REFERENCES}

Abel, T., Bryan, G. L., \& Norman, M. L. 2000, ApJ, 540, 39 -. 2002, Science, 295, 93

Abel, T., Norman, M. L., \& Madau, P. 1999, ApJ, 523, 66

Abel, T., Wise, J. H., \& Bryan, G. L. 2007, ApJ, 659, L87

Ahn, K. \& Shapiro, P. R. 2007, MNRAS, 375, 881

Ahn, K., Shapiro, P. R., Iliev, I. T., Mellema, G., \& Pen, U. 2009, ApJ, 695, 1430

Alvarez, M. A., Bromm, V., \& Shapiro, P. R. 2006, ApJ, 639, 621

Alvarez, M. A., Wise, J. H., \& Abel, T. 2009, ApJ, 701, L133

Anninos, P., Zhang, Y., Abel, T., \& Norman, M. L. 1997, New Astronomy, 2, 209

Bromm, V., Coppi, P. S., \& Larson, R. B. 1999, ApJ, 527, L5

-. 2002, ApJ, 564, 23

Bromm, V., Yoshida, N., \& Hernquist, L. 2003, ApJ, 596, L135

Cen, R. \& Riquelme, M. A. 2008, ApJ, 674, 644

Draine, B. T. \& Bertoldi, F. 1996, ApJ, 468, 269

Galli, D. \& Palla, F. 1998, A\&A, 335, 403

Greif, T. H., Johnson, J. L., Bromm, V., \& Klessen, R. S. 2007, ApJ, 670, 1

Greif, T. H., Johnson, J. L., Klessen, R. S., \& Bromm, V. 2008, MNRAS, 387, 1021

Haiman, Z., Abel, T., \& Rees, M. J. 2000, ApJ, 534, 11

Haiman, Z., Rees, M. J., \& Loeb, A. 1997, ApJ, 476, 458

Hasegawa, K., Umemura, M., \& Susa, H. 2009, MNRAS, 445

Hayes, J. C., Norman, M. L., Fiedler, R. A., Bordner, J. O., Li, P. S., Clark, S. E., ud-Doula, A., \& Mac Low, M.-M. 2006, ApJS, 165, 188

Heger, A. \& Woosley, S. E. 2002, ApJ, 567, 532

Iliev, I. T., Shapiro, P. R., \& Raga, A. C. 2005, MNRAS, 361, 405

Iliev, I. T., Whalen, D., Mellema, G., Ahn, K., Baek, S., Gnedin, N. Y., Kravtsov, A. V., Norman, M., Raicevic, M., Reynolds, D. R., Sato, D., Shapiro, P. R., Semelin, B., Smidt, J., Susa, H., Theuns, T., \& Umemura, M. 2009, MNRAS, 1313

Johnson, J. L. \& Bromm, V. 2006, MNRAS, 366, 247

Johnson, J. L., Greif, T. H., \& Bromm, V. 2007, ApJ, 665, 85

Kitayama, T. \& Yoshida, N. 2005, ApJ, 630, 675

Kitayama, T., Yoshida, N., Susa, H., \& Umemura, M. 2004, ApJ, 613,631

Machacek, M. E., Bryan, G. L., \& Abel, T. 2001, ApJ, 548, 509

-. 2003, MNRAS, 338, 273

MacIntyre, M. A., Santoro, F., \& Thomas, P. A. 2006, MNRAS, 368,1301

Mellema, G., Iliev, I. T., Alvarez, M. A., \& Shapiro, P. R. 2006, New Astronomy, 11, 374
Mesinger, A., Bryan, G. L., \& Haiman, Z. 2006, ApJ, 648, 835 -. 2009, MNRAS, 399, 1650

Mizuta, A., Kane, J. O., Pound, M. W., Remington, B. A.,

Ryutov, D. D., \& Takabe, H. 2006, ApJ, 647, 1151

Nakamura, F. \& Umemura, M. 2001, ApJ, 548, 19

-. 2002, ApJ, 569, 549

O'Shea, B. W., Abel, T., Whalen, D., \& Norman, M. L. 2005, ApJ, 628, L5

O'Shea, B. W. \& Norman, M. L. 2007, ApJ, 654, 66

—. 2008, ApJ, 673, 14

Ricotti, M., Gnedin, N. Y., \& Shull, J. M. 2001, ApJ, 560, 580

Sakuma, M. \& Susa, H. 2009, ApJ, 698, 155

Schaerer, D. 2002, A\&A, 382, 28

Shapiro, P. R., Iliev, I. T., \& Raga, A. C. 2004, MNRAS, 348, 753

Stone, J. M. \& Norman, M. L. 1992, ApJS, 80, 753

Susa, H. 2007, ApJ, 659, 908

Susa, H. \& Umemura, M. 2006, ApJ, 645, L93

Susa, H., Umemura, M., \& Hasegawa, K. 2009, ApJ, 702, 480

Tominaga, N. 2009, ApJ, 690, 526

Tominaga, N., Umeda, H., \& Nomoto, K. 2007, ApJ, 660, 516

Turk, M. J., Abel, T., \& O'Shea, B. 2009, Science, 325, 601

van Leer, B. 1977, Journal of Computational Physics, 23, 276

Vishniac, E. T. 1983, ApJ, 274, 152

Whalen, D., Abel, T., \& Norman, M. L. 2004, ApJ, 610, 14

Whalen, D. \& Norman, M. L. 2006, ApJS, 162, 281

- 2008a, ApJ, 673, 664

Whalen, D., O'Shea, B. W., Smidt, J., \& Norman, M. L. 2008a, ApJ, 679, 925

Whalen, D., van Veelen, B., O'Shea, B. W., \& Norman, M. L.

2008b, ApJ, 682, 49

Whalen, D. J. \& Norman, M. L. 2008b, ApJ, 672, 287

Williams, R. J. R. 2002, MNRAS, 331, 693

Wise, J. H. \& Abel, T. 2005, ApJ, 629, 615

-. 2007, ApJ, 671, 1559

-. 2008a, ApJ, 684, 1

-. 2008b, ApJ, 685, 40

Yoshida, N., Abel, T., Hernquist, L., \& Sugiyama, N. 2003, ApJ, 592,645

Yoshida, N., Oh, S. P., Kitayama, T., \& Hernquist, L. 2007, ApJ, 663, 687 